\title{
INCIDENCIA DEL CÁNCER UROLÓGICO EN UN ÁREA SANITARIA DE 300.000 HABITANTES
}

\author{
D. SANTOS ARRONTES, A. PÁEZ BORDA**, M. LUJÁN GALÁN, L. LLANES GONZÁLEZ, \\ C. ESCALERA ALMENDROS, C. PASCUAL MATEOS, A. BERENGUER SÁNCHEZ
}

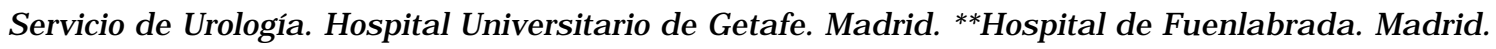

Actas Urol Esp. 28 (9): 646-649, 2004

\section{RESUMEN}

INCIDENCIA DEL CÁNCER UROLÓGICO EN UN ÁREA SANITARIA DE 300.000 HABITANTES

OBJETIVO: El objetivo de este trabajo es aportar información sobre la incidencia cáncer específica ajustada de los tumores urológicos durante un período de 10 años en el área geográfica de Getafe (Madrid, España, 300,000 habitantes).

MATERIAL Y MÉTODOS: En este estudio retrospectivo se incluyeron todos los pacientes diagnosticados histológicamente de cáncer genitourinario (vejiga, próstata, riñón, pene y testículo) entre 1992 y 2001. Todos los tumores fueron clasificados de acuerdo a las normas internacionales. Se calcularon las tasas de incidencia ajustada (estandarizada) por cada 100.000 habitantes (o por cada 100.000 varones en los casos de los tumores de próstata, pene y testículo). Además se evaluó el incremento global y cáncer específico anual. Para el ajuste de la población fueron utilizados los datos poblacionales más recientes publicados por el Instituto Nacional de Estadística.

RESULTADOS: El cáncer de próstata fue el tumor más frecuente. Se detectó una importante correlación entre el número total de nuevos diagnósticos de cáncer y el incremento de la población. Sin embargo ésta no pudo ser demostrada cuando se compararon los nuevos diagnósticos en mujeres con el incremento de población femenina, pero sí en el caso de la masculina. No obstante, únicamente el número de nuevos casos de cáncer de próstata se asoció de manera significativa con la población.

CONCLUSIONES: Las tasas de incidencia de cada cáncer se han incrementado a lo largo de la última década, sin embargo, este incremento no ha sido paralelo al incremento de la población, probablemente debido a la modificación de factores ambientales.

PALABRAS CLAVE: Incidencia. Epidemiologia. Tumores genitourinarios.

\section{ABSTRACT}

GENITOURINARY CANCER INCIDENCE IN A HEALT GEOGRAPHIC AREA OF 300.000 PEOPLE

OBJECTIVE: To provide descriptive information on site-specific urological cancer occurrence we computed 10-year cancer incidence rates in the geographic area of Getafe (Madrid, Spain, 300,000 people).

MATERIALS AND METHODS: Only histologically confirmed genitourinary cancer (bladder, prostate, kidney, testicle and penis) throughout 1992-2001 was considered. Cancers were classified according to the international rules.

10 years age-standardized population-adjusted incidence rates per 100,000 people (or per 100,000 men when appropriate) were calculated. Overall and cancer-specific yearly increments were also evaluated. The most recent release of National Statistics (2001) was used for population adjusting.

RESULTS: Prostate cancer (PC) accounted for the majority of diagnostics. A strong correlation was detected between the total number of new cancer diagnostics and the overall population. While the correlation was frail between the number of new cancer diagnostics in females and the female population, the association remained significant in males. Nevertheless, only the number of new prostate cancer diagnostics was firmly and significantly associated with the population.

CONCLUSIONS: Incidence rates of every cancer type increased throughout the last decade. Nevertheless, this increases didn't parallel the population increment perhaps translating environmental factors.

KEY WORDS: Incidence. Epidemiology. Urogenital neoplasm. 
$\mathrm{L}$ a patología tumoral constituye uno de los problemas de salud más importantes en los países desarrollados. En España fallecieron 97.714 personas debido a patología tumoral en el año 2001 (27,1\% de la mortalidad global), únicamente superado por las enfermedades cardiovasculares $^{1}$.

La determinación de la incidencia cáncer específica, la prevalencia y la mortalidad son importantes para el establecimiento de una política de planificación sanitaria, además de ser variables esenciales en los análisis coste-efectividad. Al contrario que en otros países, en España no existe un registro nacional del cáncer, pues no se trata de una enfermedad de declaración obligatoria. Por ello la estimación de la incidencia tumoral es imprecisa, debiendo ser elaboradas en cada centro hospitalario a partir de la información disponible en cada uno de ellos. Por último, los cálculos epidemiológicos elaborados por agencias internacionales están basados en datos de mortalidad, de modo que las estimaciones son, en el mejor de los casos, provisionales ${ }^{2}$.

El objetivo de este trabajo es aportar información sobre la incidencia cáncer específica ajustada de los tumores urológicos durante un período de 10 años en el área sanitaria 10 del Servicio Madrileño de Salud.

\section{MATERIAL Y MÉTODOS}

En este estudio retrospectivo se incluyeron todos los pacientes diagnosticados histológicamente de cáncer genitourinario (vejiga, próstata, riñón, pene y testículo) entre el 1 de enero de 1992 y el 31 de diciembre de 2001. Todos los tumores fueron clasificados de acuerdo a las normas internacionales ${ }^{3}$.

Se calcularon las tasas de incidencia ajustada (estandarizada) por cada 100.000 habitantes (o por cada 100.000 varones en los casos de los tumores de próstata, pene y testículo) en el área geográfica de Getafe (Madrid, España, 300.000 habitantes). Además se evaluó el incremento global y cáncer específico anual. Para el ajuste de la población fueron utilizados los datos poblacionales más recientes publicados por el Instituto Nacional de Estadística (INE) ${ }^{1}$.

Se estudió la asociación lineal entre el número de nuevos cánceres y la población global mediante un test de correlación de Pearson utilizando la aplicación SPSS v11.5 para Windows, considerándose como significativo un error $\alpha$ menor de 0,05.

\section{RESULTADOS}

A lo largo del período de tiempo a estudio la población se ha incrementado en más de 35.000 personas, lo que supone un incremento global de un 15,5\% (Fig. 1). Se diagnosticaron 1.156 tumores genitourinarios, siendo el cáncer de próstata (CaP) el más frecuente (533 nuevos casos, 46,10\% del total de nuevos cánceres). El cáncer vesical constituyó el 44,89\% de los nuevos diagnósticos (519/1159), el cáncer renal el 5,7\% (66 casos), el testicular el 2,5\% (29 casos) y por último, el cáncer de pene el $0,77 \%$ (9 casos). Las Tablas 1 y 2 reflejan los resultados de esta parte del estudio.

La tendencia al alza en el número de cánceres es evidente con un incremento anual porcentual para el cáncer de próstata de 5,09\% (con un rango entre 2,78-7,46) y para los cánceres de vejiga y riñón de $1,81 \%(-0,45-4,12)$ y de $2,79 \%$ $(-3,89$ - 9,94), respectivamente (Fig. 2).

Se detectó una importante correlación entre el número total de nuevos diagnósticos de cáncer y el incremento de la población $\left(\mathrm{r}=0,75, \mathrm{r}^{2}=0,56\right.$; $p=0,01)$. Sin embargo ésta no pudo ser demostrada cuando se compararon los nuevos diagnósticos en mujeres con el incremento de población femenina $\left(r=0,49 ; r^{2}=0,24 ; p=0,14\right)$, pero sí en el

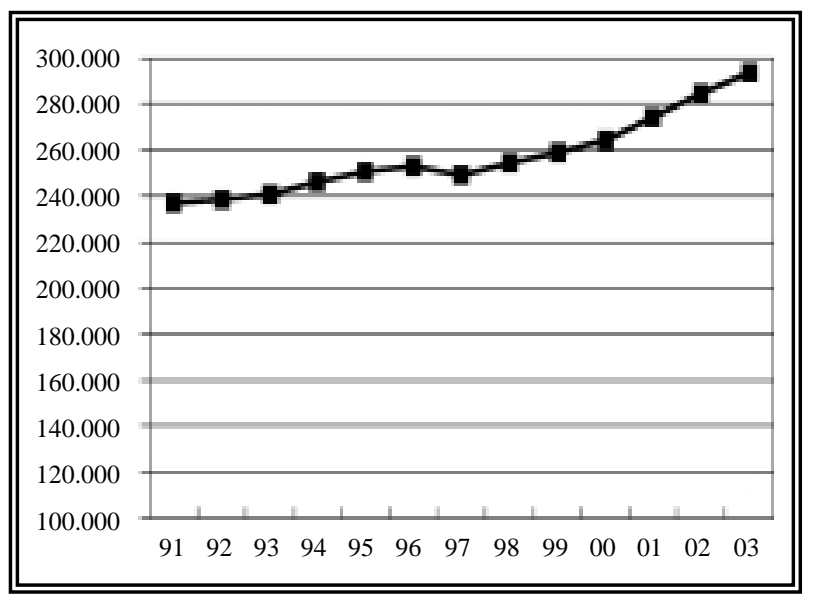

FIGURA 1. Evolución de la población del área $10 \mathrm{del}$ Servicio Madrileño de la Salud. En el eje de ordenadas se indica el número de habitantes. Fuente: Instituto Nacional de Estadistica (INE) ${ }^{1}$. 
Tabla 1

Datos de incidencia estandarizada por 100.000 habitantes.

\begin{tabular}{|c|c|c|c|c|c|c|c|c|c|c|c|}
\hline \multirow{2}{*}{ ND } & \multirow{2}{*}{ Año } & \multicolumn{2}{|c|}{ Vejiga } & \multicolumn{2}{|c|}{ Próstata*k } & \multicolumn{2}{|c|}{ Rinón } & \multicolumn{2}{|c|}{ Testículo*k } & \multicolumn{2}{|c|}{ Pene** } \\
\hline & & ND & IA & ND & IA & ND & IA & ND & IA & ND & IA \\
\hline 64 & 1992 & 36 & 14,98 & 24 & 19,90 & 2 & 0,83 & 2 & 1,66 & 0 & 0 \\
\hline 85 & 1993 & 46 & 18,77 & 35 & 28,42 & 3 & 1,22 & 1 & 0,81 & 0 & 0 \\
\hline 78 & 1994 & 36 & 14,41 & 33 & 26,28 & 7 & 2,80 & 2 & 1,60 & 0 & 0 \\
\hline 117 & 1995 & 49 & 19,45 & 57 & 45,19 & 9 & 3,57 & 2 & 1,56 & 0 & 0 \\
\hline 103 & 1996 & 50 & 20,17 & 40 & 31,57 & 7 & 2,82 & 4 & 3,23 & 2 & 1,61 \\
\hline 136 & 1997 & 73 & 28,87 & 48 & 38,69 & 10 & 3,95 & 4 & 3,17 & 1 & 0,79 \\
\hline 147 & 1998 & 69 & 26,79 & 63 & 49,68 & 9 & 3,49 & 5 & 3,89 & 1 & 0,77 \\
\hline 112 & 1999 & 45 & 17,15 & 63 & 48,73 & 3 & 1,14 & 0 & 0 & 1 & 0,76 \\
\hline 169 & 2000 & 52 & 19,82 & 101 & 76,64 & 12 & 4,57 & 4 & 3,05 & 0 & 0 \\
\hline 145 & 2001 & 63 & 24,01 & 69 & 50,36 & 4 & 1,52 & 5 & 3,81 & 4 & 2,81 \\
\hline 1156 & Total & 519 & $\mathrm{NC}$ & 533 & $\mathrm{NC}$ & 66 & $\mathrm{NC}$ & 29 & $\mathrm{NC}$ & 9 & $\mathrm{NC}$ \\
\hline
\end{tabular}

ND: Nuevos diagnósticos. IA: Incidencia ajustada. NC: No calculado. El ajuste de poblaciones realizó según los datos del INE. **Ajustada para cada 100.000 varones. Las cifras en negrita indican el año de mayor número de diagnósticos; y en cursiva, la incidencia anual mayor alcanzada por cada tumor en el período de tiempo considerado.

Tabla 2

Incidencia estandarizada (por 100.000 habitantes) para los diferentes tipos de cáncer urológico.

\begin{tabular}{lccccc}
\hline & Vejiga & Próstata** & Riñón & Testículo** & Pene*** \\
\hline Media & 20,44 & 41,54 & 2,59 & 2,27 & 0,67 \\
Error & & & & & \\
estándar & 4,77 & 16,40 & 1,32 & 1,33 & 0,92 \\
Mínimo & 14,41 & 19,90 & 0,83 & 0 & 0 \\
Máximo & 28,87 & 76,64 & 4,57 & 3,89 & 2,81 \\
\hline
\end{tabular}

**Incidencia por 100.000 varones.

caso de la masculina $\left(r=0,72 ; r^{2}=0,53 ; p=0,017\right)$. No obstante, únicamente el número de nuevos casos de CaP se asoció de manera significativa con el incremento de población $\left(r=0,72 ; r^{2}=0,53\right.$; $\mathrm{p}=0,017$ ).

\section{DISCUSIÓN}

Desafortunadamente no existen registros de cáncer a nivel universal. Teniendo esto en cuenta, los métodos utilizados para estimar las tasas de incidencia se basan en varias posibilidades, por un lado en los registros nacionales (o regionales) de incidencia, y por otro en los registros nacionales (o regionales) de mortalidad. A partir de estos datos disponibles se han elaborado estrategias que nos permitan calcular la incidencia.

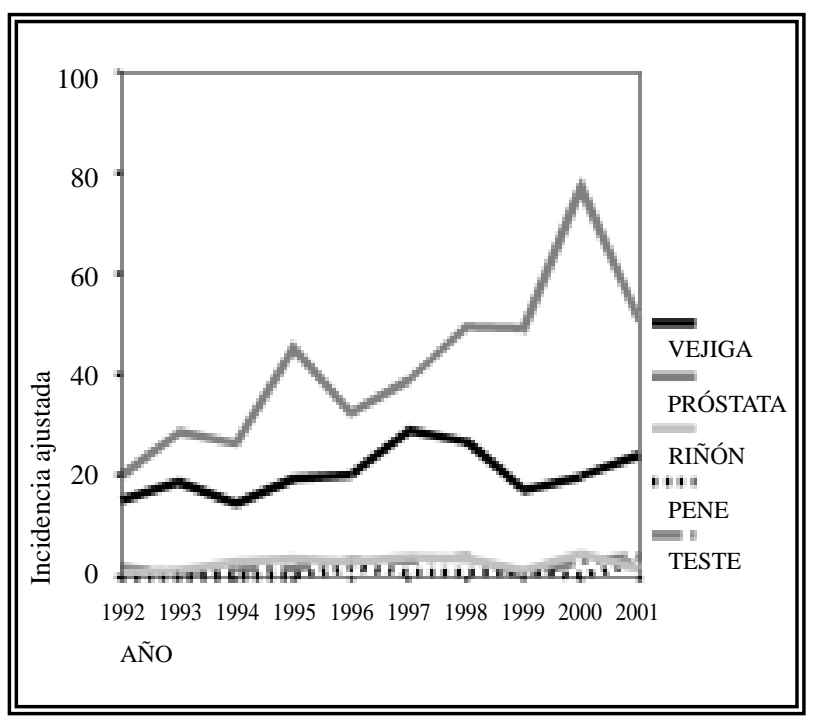

FIGURA 2. Incidencia ajustada de los diferentes tumores genitourinarios.

Dado que en España los registros de cáncer sólo engloban al $26 \%$ de la población ${ }^{4}$, el cálculo de la incidencia se basaría en el segundo supuesto, ya que sí disponemos de un registro nacional de mortalidad por cáncer, lo que nos permite calcular la incidencia usando modelos matemáticos que, además, tengan en cuenta las cifras de mortalidad e incidencia a nivel local o regional cuando estén disponibles. 
Nuestro estudio representa un buen escenario para establecer las tasas de incidencia en el área de Madrid. Nuestro hospital pertenece al Sistema Nacional de Salud (SNS), siendo el único centro existente en un área de 300.000 habitantes, por lo que la mayor parte de los test diagnósticos o biopsias son indicadas y realizadas en nuestro departamento. Además, y teniendo en cuenta: el perfil socioeconómico de la población (en su mayoría trabajadores de mediana edad, jubilados o parados), las características del SNS (universal y gratuito) y la libre utilización de marcadores tumorales por parte de los facultativos, nos hace sospechar que el número de consultas privadas fuera del ámbito hospitalario sean muy bajas. Por ello consideramos que las tasas de incidencia calculadas son una estimación aceptable, aunque los resultados deben tomarse con precaución.

Especialmente interesante es el incremento permanente en la incidencia del cáncer de próstata. Esto puede ser debido en parte al uso extensivo e indiscriminado del PSA. Esta tendencia también ha sido detectada por otro grupos ${ }^{5,6}$. Sin embargo, la incidencia actual del cáncer de próstata en España debe ser todavía mayor.

La más reciente publicación de la Agencia Internacional para la Investigación en Cáncer de la Organización Mundial de la Salud (OMS) ${ }^{2}$, hace una infravaloración del incremento de incidencia del $\mathrm{CaP}$ en España, probablemente en relación con una insuficiente determinación del uso del PSA en nuestro medio (la incidencia del CaP en España calculada por la OMS para el año 2000 fue de 24,23 casos / 100.000 varones). En una reciente revisión nuestra referente al uso del PSA en el área de Getafe se objetivó que en el grupo de edad entre 55 y 69 años se realizaron 86,8 determinaciones por cada 1.000 habitantes (152,6 en mayores de 70 años), lo que representa cientos de biopsias prostáticas y la detección de numerosos $\mathrm{CaP}^{7}$.

Por el contrario la incidencia global del cáncer vesical parece que está en una fase de "plateau". Es posible, que el descenso en el consumo de cigarrillos sea el responsable de esta tendencia ${ }^{8}$. La incidencia del resto de tumores (riñón, testículo y pene) se mantiene baja con una muy leve tendencia al ascenso a lo largo de la década (Tabla 1), en probable relación con factores ambientales y genéticos.

\section{CONCLUSIONES}

El CaP es la neoplasia urológica más frecuente en nuestro medio, mientras que en las mujeres es el cáncer vesical.

Las tasas de incidencia de cada cáncer se han incrementado a lo largo de la última década, sin embargo, este incremento no ha sido paralelo al incremento de la población, probablemente debido a la modificación de factores ambientales. La implantación de estrategias de screening probablemente justifiquen el aumento en la incidencia del CaP. La contención en el hábito tabáquico presumiblemente explique el "plateau" en la incidencia del cáncer vesical.

\section{REFERENCIAS}

1. http://www.ine.es/prensa/np313.pdf. Última entrada 20/ $2 / 2004$.

2. Agency for Research on Cancer of the World Health Organization (WHO) http://www-dep.iarc.fr/globocan/globocan.html. Última entrada 01/2/04.

3. Percy C, Van Holten V, Muir C (eds). International classification of diseases for oncology. $2^{\text {nd }}$ ed. Geneva, Switzerland: World Health Organization 1990.

4. Miñarro R, Black RJ, Martinez C. Cancer incidence and mortality in Spain: patterns and trends [Incidencia y mortalidad de cáncer en España: patrones y tendencias]. IARC technical report No. 36., international agency for research on cancer, Lyon (2000).

5. Jemal A, Murray T, Samuels A, Ghafoor A, Ward E, Thun M. Cancer statistics 2003. CA Cancer J Clin 2003;53:5-26.

6. A. Quaglia A, Parodi S, Grosclaude P, Martínez-García C, Coebergh JV, Vercelli M. Differences in the epidemic rise and decrease of prostate cancer among geographical areas in Southern Europe. An analysis of differential trends in incidence and mortality in France, Italy and Spain. Eur J Cancer 2003;39(5):654-665.

7. Páez A, Luján M, Llanes L, Romero I, de la Cal MA, Miravalles E, Berenguer A: Assessing trends of PSA-use. Eur Urol 2002;41:162-166.

8. Prevención y Promoción de la Salud. Ministerio de Sanidad y Consumo. http://www.msc.es/salud/epidemiologia/ tabaco. Última entrada 26/Enero/2004.

Dr. D. Santos Arrontes

Hospital Santa Bárbara

Ctra. de Malagón, s/n

13500 Puertollano (Ciudad Real)

(Trabajo recibido el 16 marzo de 2004) 